\title{
L'impensé pédagogique du Télémaque de Fénelon
}

\section{Alain Vergnioux}

\section{(2) OpenEdition \\ 1 Journals}

\section{Édition électronique}

URL : https://journals.openedition.org/dhfles/1519

DOI : $10.4000 /$ dhfles. 1519

ISSN : 2221-4038

Éditeur

Société Internationale pour l'Histoire du Français Langue Étrangère ou Seconde

Édition imprimée

Date de publication : 1 juin 2003

Pagination : 33-45

ISSN : 0992-7654

\section{Référence électronique}

Alain Vergnioux, «L'impensé pédagogique du Télémaque de Fénelon », Documents pour l'histoire du français langue étrangère ou seconde [En ligne], 30 | 2003, mis en ligne le 01 janvier 2012, consulté le 27 mai 2021. URL : http://journals.openedition.org/dhfles/1519; DOI : https://doi.org/10.4000/dhfles. 1519

Ce document a été généré automatiquement le 27 mai 2021.

(c) SIHFLES 


\title{
L'impensé pédagogique du Télémaque de Fénelon
}

\author{
Alain Vergnioux
}

1 Que les qualités, les caractéristiques linguistiques et littéraires du Télémaque aient été un puissant levier pour sa diffusion et son usage pédagogique, les communications présentées à ce Colloque en fournissent la démonstration convaincante.

Dès sa publication en 1699 , et en dépit des attaques dont il fut l'objet ${ }^{1}$, le succès du roman de Fénelon fut en effet immédiat.

On compte soixante-quinze éditions françaises au XVIII ${ }^{e}$ siècle, mais l'engouement est à son comble au début du XIX ${ }^{\mathrm{e}}$ : deux cent dix éditions entre 1800 et 1850 , sans compter les réimpressions ; soixante sept entre 1850 et $1900^{2}$. Dès le début du siècle, le livre est traduit en allemand (vingt-huit éditions allemandes entre 1700 et 1888), en anglais (trente trois éditions anglaises entre 1725 et 1837), en italien (trente et une éditions entre 1704 et 1841), etc. Notons aussi tout au long du XVIIIe siècle des traductions en espagnol, portugais, grec, arménien, suédois, hollandais, hongrois, polonais, sans oublier, entre 1837 et 1845, trois éditions polyglottes en six langues (français, anglais, allemand, italien, espagnol, portugais). Cette énumération, qui pourrait être amplement poursuivie, souligne un premier trait : la capacité du texte à assurer entre les langues un rôle de médiation - ce que l'expérience de Jacotot poussera à sa limite : le Télémaque comme point de départ d'une méthode d'éducation universelle.

Nous voudrions cependant proposer pour cet extraordinaire succès une série d'hypothèses différentes que nous rassemblons sous la notion d'impensé et que l'on peut décrire à travers quatre inversions.

La première inversion concerne l'utopie politique portée par le texte ; la seconde concernerait l'ambivalence sexuelle de Mentor ; la troisième réside dans le thème du meurtre du fils par le père et la dernière porte sur l'usage que fait du texte Joseph Jacotot dans son projet d'Enseignement universel (1823). 


\section{Inversion 1 : L'utopie politique portée par le texte : République versus Monarchie}

\section{La figure du bon roi}

Dans le roman, Mentor dresse à plusieurs reprises pour son élève des portraits de rois exemplaires : Sésostris au Livre II, Minos au Livre V, Idoménée (après son repentir) au Livre $\mathrm{X}$, montrant chaque fois les conséquences heureuses de leur conduite dans les domaines de la paix, du bien-être du peuple, de la richesse économique et de la gloire du souverain. Les principes de cette éducation sont la simplicité des moeurs, la retenue, la pudeur, le dévouement au bien commun qui doit passer avant les intérêts égoïstes et l'amour de soi. Le prince doit savoir se montrer juste et fort, être respecté et aimé de ses sujets parce que toute son action vise à assurer leur bonheur. Heureux « le roi qui fait le bonheur de son peuple et qui trouve son bonheur dans la vertu. Il est plus que craint car il est aimé. Non seulement on lui obéit, mais encore il est le roi de tous les coeurs » (Livre II : 29).

7 Le bon souverain doit savoir écouter son peuple et exercer le pouvoir avec un esprit de justice, à l'exemple de Sésostris, roi de Thèbes :

Il écoutait chaque jour à certaines heures réglées, tous ceux de ses sujets qui avaient des plaintes à lui faire ou des avis à lui donner. Il ne méprisait ni ne rebutait personne et ne croyait être roi que pour faire du bien à tous ses sujets (ibid. : 31).

8 À l'inverse, c'est par les conséquences désastreuses de leurs conduites que Mentor veut dissuader Télémaque de suivre les voies adoptées par les «mauvais» rois. Ainsi de Pygmalion, roi des Phéniciens, tellement assoiffé de pouvoir qu'il fit son propre malheur en même temps qu'il conduisit son peuple à la ruine.

Tourmenté par une soif insatiable de richesses, [il] se rend de plus en plus misérable et odieux à ses sujets [...]. L'avarice le rend défiant, soupçonneux, cruel [...]. Tout l'agite, l'inquiète, le ronge ; il a peur de son ombre, il ne dort ni nuit ni jour [...] ; il est seul, triste, abattu au fond de son palais [...] ; il ne connait ni les doux plaisirs, ni l'amitié encore plus douce [...]. Il ne peut cacher les remords qui déchirent ses entrailles ; les mets les plus exquis le dégoûtent (Livre III : 56-57).

9 À travers rencontres et expériences, ainsi, Télémaque mûrit. Il apprend à dominer ses impulsions, il se montre plus réfléchi, raisonne avec son maître. Les aventures qui le mènent de son naufrage en Sicile à la Phénicie puis à Chypre, de son emprisonnement en Égypte à son séjour en Crète et à Salente auprès d'Idoménée qu'il seconde dans la guerre contre les Manduriens, lui font pénétrer les difficultés et les dangers du pouvoir, mais lui montrent aussi la joie et la fierté que procure le succès s'il s'appuie sur la raison et la justice. De même ses forces physiques grandissent et il en fait usage à bon escient jusqu'à devenir un parfait chef de guerre.

Mais la liberté d'une nation et le bonheur de ses habitants supposent la paix et la prospérité. Aussi l'éducation du prince comporte-t-elle un volet économique et un autre de politique étrangère.

11 La richesse d'une nation repose sur l'agriculture et le commerce. Sur ce dernier point, Mentor prône la liberté des échanges et donne en exemple au Livre III le modèle des Phéniciens : " recevez bien et facilement tous les étrangers ; faites leur trouver dans vos ports la sûreté, la commodité, la liberté entière » (64). Et, quand, au Livre X, ils accompagnent Idoménée dans la visite du port de Salente, Mentor énonce des règles 
pour le contrôle de la banque et en faveur de la liberté du commerce, qu'il ne faut pas gêner " par des impôts ». " Le commerce de cette ville était semblable au flux et reflux de la mer. Les trésors y entraient comme les flots viennent l'un sur l'autre. Tout y était apporté et tout en sortait librement ; tout ce qui entrait était utile, tout ce qui sortait laissait en sortant d'autres richesses en sa place » (264).

Il faut encourager l'artisanat, mais non les productions de luxe qui corrompent les mœurs. Pour promouvoir son développement, il convient de former de bons ouvriers. Ainsi à Tyr :

on traite avec honneur tous ceux qui réussissent dans les arts [...] ; on ne méprise point un bon charpentier, au contraire, il est bien payé et bien traité [...], et le père est ravi d'élever son fils dans un si bon métier (67).

Quant à faire l'éloge de l'agriculture, le récit en offre maintes fois l'occasion, mais en particulier, quand ils arrivent en Crète dont Minos assure la sage administration, ils découvrent une campagne

fertile et ornée de tous les fruits par le travail de ses habitants [...] ; nous ne trouvions aucun champ où la main du diligent laboureur ne fût imprimée ; partout la charrue avait laissé de creux sillons [...] ; nous considérions avec plaisir les creux vallons où les troupeaux de bœufs mugissaient dans les gras herbages le long des ruisseaux (100).

14 Les idées politiques de Fénelon semblent ainsi prises dans un entre-deux. Dans le Télémaque, il admet le principe d'une royauté de droit divin - comment pourrait-il en être autrement puisqu'il éduque un futur roi ? Mais les descriptions qu'il en donne, expriment à cet égard un certain nombre de principes : le roi est « l'homme du peuple ", « l'homme des lois et l'homme de Dieu » : c'est à l'intérieur de ce triangle qu'il assoit son autorité. Ce qui doit le guider, c'est la charité, l'amour du peuple, la loi naturelle qui est la source des autres lois. Cette thèse, laïcisée, verra dans la Raison la source et la loi de tout gouvernement. Dans cette perspective, le relais dans le récit avec la pensée politique du XVIII ${ }^{e}$ est assuré par la figure de Minerve.

Dans les Tables de Chaulnes et les écrits politiques des années 1700, Fénelon développe sur l'organisation concrète du royaume des idées réformatrices soucieuses de modérer l'absolutisme royal : il affirme que le pouvoir du roi doit être limité par les États généraux, dont l'indépendance est garantie : ils votent les impôts et contrôlent leur levée, délibèrent sur la justice, le commerce, la guerre et la paix ; l'administration royale des provinces doit être rendue plus homogène et les Parlements rétablis dans leurs droits. Selon Roland Mousnier, les Tables de Chaulnes veulent établir " un socialisme d'État, agraire et chrétien " (1976: 114), que l'on trouve aussi dans la description de Salente où le roi apparaît comme le premier magistrat d'une cité où tous sont égaux - vision chimérique au regard de la société française du XVII ${ }^{e}$ siècle.

\section{Paix et cosmopolitisme}

Les principes de politique internationale se trouvent en substance exprimés quand Télémaque, à l'occasion du concours organisé pour désigner le successeur de Minos (Livre V), doit répondre à la question : lequel des deux rois est préférable, le conquérant invincible à la guerre ou le roi « sans expérience de la guerre mais propre à policer sagement les peuples dans la paix " ? Télémaque, contre l'avis général, fait l'éloge du roi pacifique (119). «S'il est véritablement propre à gouverner en paix, il a toutes les qualités nécessaires pour mettre son peuple en sûreté contre ses ennemis »; 
il est « juste, modéré et commode avec ses voisins ", « fidèle » dans ses alliances ; sa " probité, sa bonne foi » en font un arbitre tout désigné pour régler les litiges. Protégé derrière ses frontières, le peuple prospère et croît en nombre, prêt à se battre avec courage à la moindre menace, car ils « aimeront mieux mourir que de passer sous la domination d'un autre roi, violent et injuste» (121) ; enfin, le souverain pacifique n'aura pas de peine, s'il est menacé, à trouver de nombreux alliés pour le soutenir.

En politique extérieure, Fénelon ne manque donc pas de hardiesse ; il ne se contente pas de condamner avec vigueur toutes les guerres et avant tout les guerres de conquête, il prône la conciliation et l'arbitrage, croit en la puissance de la diplomatie pour résoudre les conflits, de telle sorte qu'un Charles Dédéyan peut souligner son cosmopolitisme (Dédéyan 1991 : chap. 6) : il est présent dans la géographie du Télémaque : de la Phénicie à la Grèce, de l'Égypte à Chypre les peuples commercent pour leur prospérité commune. Mais dans un de ses derniers textes, l'Examen de conscience sur les devoirs de la royauté, édité après sa mort en 1734, Fénelon nous livre sur ce sujet sa profession de foi :

Toutes les Nations de la terre ne sont que les différentes familles d'une même République dont Dieu est le père commun. La loi naturelle et universelle, selon laquelle il veut que chaque famille soit gouvernée, est de préférer le bien public à l'intérêt particulier (in Dédéyan 1991 : 332).

Cette première inversion pourrait ainsi s'énoncer : l'éducation du bon roi s'inverse en programme républicain. Voltaire ne s'y était pas trompé qui voyait dans le Télémaque " un pamphlet révolutionnaire et une agréable fiction politique ».

\section{Inversion 2 : l'ambivalence sexuelle de Mentor et l'érotisme latent qui parcourt le texte}

\section{Les figures féminines}

Sans doute le moteur du récit est-il la recherche par Télémaque de son père, sans doute le jeune homme rencontre-t-il, pour son instruction et sa formation de nombreux modèles masculins mais le récit est largement occupé aussi par des figures féminines. Indiquons tout de suite leur fonction médiatrice.

Tenons-nous en à la phrase inaugurale : "Calypso ne pouvait se consoler du départ d'Ulysse ». Fonction de médiation dans l'absence. Absence d'Ulysse certes, absent pour Télémaque qui le recherche - mais davantage ici pour Calypso son amante abandonnée. Les trajectoires respectives du père et du fils font en effet qu'ils ne se rejoindront jamais, que toujours ils se manquent. Dans ce moment inaugural de l'arrivée dans l'île, d'abord, et pour cause : le fils vient prendre la place du père dans le lit de la déesse; à la fin du récit encore, au livre XVIII, quand ils se croisent dans une petite île proche d'Ithaque où Télémaque a dû relâcher faute de vent, avant de pouvoir terminer son voyage, c'est-à-dire, auprès de Pénélope : la mère / épouse inaccessible 3 .

J'avancerai cette thèse : il ne peut $\mathrm{y}$ avoir de médiation directe entre le père et le fils sinon par le biais des femmes, d'un côté Calypso, l'amante, de l'autre la déesse Minerve, la mère institutrice, dont Mentor est un avatar ambigu. La mère oedipienne, Pénélope, n'apparaîtra jamais. Et puis enfin Eucharis qui vient troubler le jeu. 

bannis, le désir et la séduction trouvent des formes d'expression discrètes mais troublantes, faites de grâce et d'élégance : "Télémaque suivait la déesse environnée d'une foule de jeunes nymphes [...]. Il admirait l'éclat de sa beauté, la riche pourpre de sa robe longue et flottante, ses cheveux noués par derrière négligemment mais avec grâce, le feu qui sortait de ses yeux » (6). À quoi fait écho toute une sensualité de la nature, comme la description de la grotte en porte témoignage : " elle était tapissée d'une jeune vigne qui étendait ses branches souples également de tous côtés. Les doux zéphyrs conservaient en ce lieu, malgré les ardeurs du soleil, une délicieuse fraîcheur : des fontaines coulant avec un doux murmure sur des prés semés d'amarante et de violettes formaient en divers lieux des bains aussi purs et aussi clairs que le cristal : mille fleurs naissantes émaillaient les tapis verts dont la grotte était environnée " (ibid.). Ou plus loin : " Alors on s'assit sur l'herbe semée de violettes, à l'ombre d'un bocage épais. Calypso ne pouvait s'empêcher de jeter sans cesse des regards tendres et passionnés sur Télémaque [...], baissant les yeux et rougissant avec beaucoup de grâce " (80). Poussons plus loin : chez Fénelon, la sensualité est une sensualité du style, et son registre est de part en part celui de la séduction. emporter son adhésion? 
Fénelon ainsi mêle les registres. Tantôt, s'impose dans la bouche de Mentor un moralisme sévère : " le naufrage et la mort sont moins affreux que les plaisirs qui attaquent la vertu. [...] Gardez-vous d'écouter les paroles douces et flatteuses de Calypso, qui se glisseront comme un serpent sous les fleurs ; craignez le poison caché " (10). Tantôt, il fait appel chez son jeune élève à une sorte de sens inné du bien et de la raison : " Je suis content de vous : vous avez fait de grandes fautes; mais elles vous ont servi à vous connaître et à vous défier de vous même » (476). Mais l'expérience de soi et du monde est aussi guidée par une instance supérieure - dans la fable, c'est l'influence de Minerve :

vous avez fait de grandes choses ; mais avouez la vérité, ce n'est guère vous par qui elles ont été faites : n'est-il pas vrai qu'elles vous sont venues comme quelque chose d'étranger qui était mis en vous ? [...] Ne sentez-vous pas que Minerve vous a comme transformé en un autre homme au dessus de vous- même ? (477).

Dans la perspective propre de Fénelon, l'idée d'un "maître intérieur » renvoie à la doctrine de l'œuvre de Dieu en nous ${ }^{6}$. Enfin, les dernières pages du livre XVIII révèlent au jeune homme, dans un éblouissement, l'essence divine et femme de son maître.

Ses yeux creux et austères se changent en des yeux bleus d'une douceur céleste et pleins d'une grâce divine [...]. Il reconnaît un visage de femme, avec un teint plus uni qu'une fleur tendre et nouvellement éclose au soleil : on y voit la blancheur des lis mêlés de roses naissantes : sur ce visage fleurit une éternelle jeunesse, avec une majesté simple et négligée [...] (531).

Le romancier pouvait dans les mêmes termes décrire la belle Eucharis ou la chaste Antiope, mais la déesse les surpasse - elle seule est en droit digne de l'amour le plus élevé auquel un mortel puisse atteindre.

31 Minerve, à la fois mère et amante inaccessible, qui trouve dans la théologie piétiste son pendant avec la figure de la vierge, mère du Christ. Ainsi Minerve peut-elle, dans les dernières pages du récit (quand est achevée l'initiation de Télémaque) s'adresser à lui en ces termes troublants : il faut maintenant « vous accoutumer à être privé de cette douceur, comme on sèvre les enfants [...] ». « Ma sagesse ne vous quittera point, pourvu que vous sentiez toujours que vous ne pouvez rien sans elle» (534).

\section{Ambivalences}

Quatre figures féminines : Calypso, Eucharis, Antiope, Minerve et une absente Pénélope dont les précédentes sont des substituts ou des variations.

Quatre figures masculines, également substitutives de la figure paternelle : Narbal, chef de la flotte phénicienne qui le sauve des Egyptiens ${ }^{7}$, Idoménée ${ }^{8}$ roi de Salente qui veut lui donner sa fille pour épouse, Termosiris, prêtre d'Apollon, qui lui prête secours quand il est prisonnier dans le désert d'Égypte ${ }^{9}$, Mentor enfin - dont la nature double assure la jonction entre les séries.

34 L'ambivalence - ou la nature double - de Mentor a été souvent soulignée ; mais il ne suffit pas de s'en tenir au dédoublement Mentor / Minerve comme à un dédoublement fonctionnel : d'un côté le pédagogue sévère, de l'autre la protectrice indulgente ; d'un côté les conditions empiriques de l'éducation, où se mêlent épreuves, erreurs et succès dans les apprentissages, de l'autre les principes de la raison et la conscience divine du bien. Il faut resituer cette ambivalence dans l'ensemble des médiations qu'elle commande : les deux séries indiquées qui constituent autant de degrés vers la sagesse 
amoureuse et la vertu politique d'une part, et les croisements qui s'effectuent entre elles d'autre part. Dans chaque cas, il ne peut y avoir de relation directe ni avec la figure de la mère ni avec celle du père, mais seulement médiatisée par la série inverse. Télémaque ne peut avoir de relation avec son père que par la médiation des femmes Calypso principalement, et de relation avec sa mère que par celle des hommes Mentor.

On pourrait trouver un commentaire de cette organisation structurale dans la théologie piétiste, et la doctrine de l'amour pur (cf. infra).

\section{Inversion 3 : le meurtre du fils par le père}

Parmi les pères de substitution, l'un intervient de façon plus insistante, du livre $\mathrm{V}$ au livre XIII, et de façon plus étrange, ou plus énigmatique. Il s'agit d'Idoménée dont la figure articule la relation filiale sur un mythe archaïque, inverse de la description freudienne : le meurtre du fils par le père.

Nous sommes au livre V. Idoménée, au retour de la guerre de Troie et assailli par la tempête, avait fait à Neptune le serment, s'il lui accordait le salut, de lui immoler « la première tête " qui se présenterait à ses yeux après qu'il aurait touché terre. Sa demande est exaucée ; il échappe aux dangers de la mer et peut regagner les rivages de sa patrie, mais le premier être qui se présente et « se jette à son cou » est son propre fils, qui s'offrira content à l'épée de son père : « me voici mon père ; votre fils est prêt à mourir pour apaiser le dieu [...]. Frappez mon père ; ne craignez point de trouver en moi un fils indigne de vous " (107). Idoménée le transperce de son épée, après quoi, dans l'excès de sa douleur, il s'enfuit sur un vaisseau pour fonder un nouveau royaume, le royaume de Salente, où Mentor et Télémaque aborderont plus loin dans le récit, au livre XIII.

La fondation et la constitution de cette nouvelle cité représentent alors les efforts du père pour se laver de sa faute en se vouant au bonheur des hommes. Fénelon va s'étendre sur le séjour de ses héros à Salente, pour y dépeindre, à travers l'échec d'Idoménée, le projet d'une cité modèle et développer toute une série de réflexions sur l'exercice du pouvoir et les conditions requises pour faire vivre une société juste et prospère. Si l'utopie d'une société bienheureuse, en un sens, s'inverse, c'est que cette fondation repose, dans son horizon, sur un crime innommable. Et, pour ajouter au malaise, soulignons que, dès leur arrivée sur l'île, Idoménée qui a reconnu ses hôtes, s'adresse à Télémaque en l'appelant " mon cher fils » tandis que la visite de l'île entremêlera l'évocation de ses malheurs à l'exaltation de la cité nouvelle ${ }^{10}$.

Ainsi le discours de la pédagogie, de la morale et de la juste conduite des affaires humaines se met en place sur fond de meurtre, le meurtre de l'enfant innocent. Au fils sacrificiel se substitue le fils adoptif. Le père pédagogue remplace le père criminel. À la filiation par le sang se substitue la filiation symbolique. À l'espace archaïque de la violence intergénérationnelle succède l'espace de la raison, de l'économie politique et de l'éducation. En outre, dans la perspective développée par Mentor, le crime d'Idoménée devient - de façon paradigmatique - la faute de tous les rois dont l'autorité est sans cesse menacée de se changer en violence et en cruauté. D'où peut alors venir la réparation? Du bon fils : ici de Télémaque. Dans le retrait du père (Ulysse) la relation éducative se met en place sous la forme d'une relation « filiale » substitutive, selon un double déplacement. Dans la fiction, la figure paternelle se dédouble en celle de Mentor 
/ Minerve, guide tout puissant, maitre et déesse, homme et femme, double accoucheur d'un futur roi. Dans un second dédoublement correspondant aux intentions éducatives de Fénelon, le Dauphin doit venir « réparer » les erreurs d'un Louis XIV à l'égard duquel Fénelon ne ménage pas les critiques, tant pour les fastes de la cour que sur les guerres menées à l'extérieur.

Mais il y a plus. On ne peut réduire l'étrange récit du crime d'Idoménée à la simple reconduction par Fénelon de thèmes empruntés au corpus mythologique de l'Antiquité. On ne peut en élucider complètement le sens sans un dernier détour vers les convictions théologiques de Fénelon, en particulier la doctrine de l'amour pur : sans espoir de récompense ni crainte d'aucun châtiment, c'est un amour parfait dont la passion du Christ offre l'image : celle d'un fils tel que voué à la mort et abandonné sur la croix, il demande la mort dans la fidélité de cet amour (ce que le fils d'Idoménée, à la rencontre de son destin, répète exactement).

On peut donc en déduire que la vision de l'amour parfait du fils implique chez le père une volonté de mort - ce que la théologie ne peut énoncer directement, car le père, aussi, est amour. La théologie du sacrifice doit ainsi concilier l'inconciliable ; l'issue classique - mais qui ne va pas sans perversité - est que le consentement de la victime comble le père.

Mais on peut suggérer aussi que l'agencement narratif de Fénelon ouvre une autre voie qui entrelace deux textes, celui de la déréliction et de la folie et celui de l'utopie raisonnable. Peut-être que sur ce point, le Télémaque assure la transition entre la pensée religieuse $\mathrm{du} \mathrm{XVII}{ }^{e}$ siècle et une certaine théologie, essentiellement morale, des Lumières.

\section{Inversion 4 : L'enseignement universel}

Encore un détour ici, auquel nous invite Joseph Jacotot, et son projet à la fois génial et délirant d'enseignement universel.

Rappelons rapidement les circonstances ${ }^{11}$. Joseph Jacotot, né en 1770, un des esprits les plus brillants de la Révolution et de l'Empire, quitte la France en 1815 pour s'installer en Belgique, où il cherche du travail, comme mathématicien, mais sans succès, jusqu'à ce qu'il trouve un poste de " lecteur pour la littérature française à l'Université de Louvain » en 1818. La difficulté qu'il rencontre est la suivante : la grande majorité de ses étudiants ne parlent que le flamand et lui ignore tout du hollandais.

La solution est à la fois théorique et empirique. Sur le premier point, l'expérience des " cours révolutionnaires ", cours accélérés et fondés sur les capacités des élèves, soutient le projet qui va se mettre en place. Sur le plan empirique, les biographes ou commentateurs s'accordent pour dire que c'est le hasard qui mit entre les mains de Jacotot une édition bilingue franco-hollandaise du Télémaque de Fénelon.

Dès lors, il s'engage dans une entreprise un peu folle. Il demande aux élèves d'apprendre par cœur le texte français, et, par confrontation avec le texte hollandais d'en deviner le sens (mot par mot) et d'en construire la grammaire. De la réussite de cette expérience sortira l'idée d'une méthode d'Enseignement Universel, i.e. qui puisse porter sur tout type de question et de matière et qui puisse s'appliquer sans distinction à tous les hommes. 

point de départ, Les aventures de Télémaque, ou tout autre livre. L'hypothèse de l'enseignement universel est que le point de départ est (déjà) un tout, le tout, et que la totalité du savoir peut être gagnée, acquise, agrégée, par ajouts, explorations, embranchements successifs. Que le moteur de la connaissance est dans son point de départ. Or il se trouve que le roman de Fénelon - d'un point de vue épistémologique permet le basculement. Construit, voire rédigé, par ajouts successifs, il est aussi conçu comme un tout : le Dauphin doit y trouver l'essentiel de son éducation. Mais le livre, s'il est une «île» - conçu pour un destinataire unique - est aussi un concentré de l'expérience humaine, dans le temps et dans l'espace. Un centre encyclopédique, auquel fait pendant une des prémisses essentielles de Jacotot : le postulat d'égalité des intelligences.

a alors dans l'expérience de Louvain un triple renversement. En premier lieu, l'expérience d'un livre clos sur lui-même qui est aussi le livre du monde, le point de départ d'une encyclopédie universelle. Ensuite, un ouvrage conçu de façon privée pour l'enseignement d'un seul, dans une perspective monarchique, devenant l'étendard méthodologique et principiel de deux principes révolutionnaires : l'égalité des intelligences et l'enseignement universel dans la perspective de l'émancipation des peuples, intellectuelle et politique. Enfin, dernier renversement, le plus scandaleux : on peut enseigner ce qu'on ignore.

49 Il y a là certes une utopie, mais on peut concevoir qu'elle se trouve dans une correspondance isomorphique avec l'utopie fénelonienne - pour peu que l'on accepte que cette dernière porte en elle les conditions de son renversement : suprématie de la raison, principe cosmopolitique.

\section{Pour conclure}

50 L'œuvre reste énigmatique, paradoxale dans sa diffusion, sa réception, dans ses interprétations et ses usages. Le charme qu'elle exerce, son influence, considérable, peuvent être rapportés à quelque chose comme son impensé : que nous avons essayé de décliner en quatre registres successifs. Sa puissance de fascination, pour reprendre un concept de Roland Barthes, pourrait être liée à sa fonction de punctum. Point de concentration, extrêmement précis - qui se détache des circonstances de son effectuation, pour acquérir un statut d'atemporalité.

51 Atemporalité, justement, qui devait permettre les interprétations à venir chez les démocrates puis les républicains du XVIII ${ }^{e}$ et $d u X X^{e}$ siècles : en plein cœur de la Révolution, le 9 février 1793, quelques jours après l'exécution de Louis XVI, le dramaturge Marie-Joseph Chénier fait représenter un Fénelon, tragédie en cinq actes où l'archevêque de Cambrai est présenté comme un précurseur des Lumières, et il n'hésitera pas à le désigner plus tard, dans ses Réflexions, comme " philosophe et patriote " (Chénier 1827 : IV, 207). Quant à Chateaubriand, dans son Essai sur les Révolutions, il se livre à une comparaison entre Platon, Fénelon et Rousseau, pour établir un parallèle entre la République, le Télémaque et l'Émile : "Platon dans sa République, Fénelon dans son Télémaque, Jean-Jacques dans son Émile, ont cherché l'homme moral et politique »(Chateaubriand $1868:$ XI, 235). 
Puissance du désir, encore, qui porte jusqu'à Louis Aragon, qui ne s'y est pas trompé, les séductions de la belle Eucharis - et celles non moins troubles de Mentor...

\section{Bibliographie}

ARAGON, Louis (1966), Les aventures de Télémaque, [Sarrebrück 1919, Paris 1920], Paris, Gallimard.

CHÉNIER, M.-Joseph (1827), CEuvres, Paris, Guillaume, t. IV, « Réflexions ».

CHATEAUBRIAND, René de (1868), Euvres complètes, Paris, Vivès.

DÉDÉYAN, Charles (1991), Télémaque ou la liberté de l'esprit, Paris, Nizet.

FÉNELON (1931), Les Aventures de Télémaque, éd. Albert Cahen, Paris, Hachette.

------(1991), Examen de conscience sur les devoirs de la royauté, in Dédéyan, Appendice XVI.

LANAVÈRE, Alain (dir.) (1976), Je ne sais quoi de pur et de sublime... Télémaque, Orléans, Paradigme.

LE BRUN, Jacques (1976), « Fénelon. Un fils est tué », in Lanavère (dir.), 193-205.

MOUSNIER, R. (1976), « Les idées politiques de Fénelon », in Lanavère (dir.).

RANCIÈRE, Jacques (1987), Le maître ignorant, Paris, Fayard.

VERMEREN, Patrice (1985), « Rien n'est dans rien ou tout le monde sait la logique », in Les sauvages dans la cité. Auto-émancipation des peuples et instruction des prolétaires au XIXe siècle, Seyssel, Champ Vallon, 76-90.

\section{NOTES}

1. Très vite la critique littéraire déclencha contre le livre de Fénelon des attaques en règles. Les premières furent le fait de Guendeville qui fit paraître entre 1700 et 1702, une Critique de mille deux cents pages où il soulignait la monotonie du récit et ses répétitions, et surtout de Faydit, auteur d'une Télémacomanie souvent hargneuse mais touchant parfois juste quand il dénonce dans l'écriture du roman son caractère guindé et ses invraisemblances (Télémacomanie, ou censure et critique du roman intitulé Les Aventures de Télémaque, fils d'Ulysse, ou suite du quatrième livre de l'Odyssée d'Homère, 1700).

2. Encore sont-ce les chiffres que l'on trouve dans le Catalogue général des livres imprimés de la Bibliothèque Nationale, 1912, tome L. La réalité est sans doute supérieure : Fleischer, en 1811, dans son Dictionnaire de la bibliographie française, dénombre pour la seule période 1761-1811, cent quinze éditions françaises et soixante quinze traductions.

3. En fait, ils se rencontrent dans deux autres circonstances, mais dans des situations limites : aux enfers, par le biais d'Arcésius, son arrière grand-père et dans un jeu de ressemblances : « Il reconnut dans le visage d'Arcésius une grande ressemblance avec Laërte ; il croyait même se 
ressouvenir confusément d'avoir vu en Ulysse, son père, des traits de cette même ressemblance " (414). La deuxième circonstance, également à la limite, se concentre de façon lapidaire dans la dernière phrase du récit : « [il] arriva à Ithaque et reconnut son père chez le fidèle Eumée » (535).

4. «Tous les mots sont zéros » (Aragon $1966: 22$ ).

5. Cf. la communication de Dora Bienaimé Rigo.

6. «Pour moi, mon Dieu, je vous trouve partout : au dedans de moi-même, c'est vous qui faites tout ce que je fais de bon. [...] C'est vous qui donnez cette volonté, c'est vous qui la conservez pure » (Instruction sur la connaissance de Dieu, cité par Cahen in Fénelon 1931 : 477).

7. «Je sens même que les dieux, que j'ai toujours servis, vous aiment et qu'ils veulent que je vous aime aussi comme mon propre fils» (53).

8. Qui l'accueille par ces mots : « Oui, vous êtes le fils d'Ulysse ; mais vous serez aussi le mien. Ô mon fils, mon cher fils!» (201).

9. «Bientôt il m'aima tendrement et me donna des livres pour me consoler. Il m'appelait : mon fils. Je lui disais souvent : mon père, les dieux qui m'ont ôté Mentor, ont eu pitié de moi : ils m'ont donné en vous un autre soutien » (38).

10. Cette analyse sera utilement complétée par la lecture de Le Brun 1976.

11. Cf. pour de plus amples développements, la contribution de Javier Suso López « Télémaque, au cœur de la méthode Jacotot » dans ces mêmes Actes.

12. Voir aussi Vermeren 1985.

\section{RÉSUMÉS}

Le succès inouï du roman de Fénelon tout au long du XVIIIe siècle et au delà, à travers des centaines d'éditions et de traductions, alors que le texte a été composé pour le seul Duc de Bourgogne, pose une sorte d'énigme que l'on tentera d'élucider à partir de l'hypothèse de l'inversion. La première inversion concerne l'utopie politique portée par le texte ; la seconde concernerait l'ambivalence sexuelle de Mentor et le croisement des deux lignes, féminine et masculine ; la troisième renverse la thèse freudienne et réside dans le thème du meurtre du fils par le père ; la dernière porte sur l'usage que fait du texte Joseph Jacotot dans son projet d'enseignement universel en 1823.

Fénelon's novel was composed for the Duke of Burgundy only. However it reached an extraordinary success throughout the 18th and further thanks to the hundred editions and translations. This fact posits an enigma of sorts that we will attempt to clarify starting from the hypothesis of inversion. The first inversion concerns the political utopia brought about in the text ; the second one consists of Mentor's sexual ambivalence of Mentor and the crossing of the two lines, the feminine and the masculine ; the third one challenges the Freudian thesis and is related to the theme of the son's murder by the father; the last one deals with the universal teaching project of Joseph Jacotot in 1823. 
INDEX

Mots-clés : ambivalence sexuelle, enseignement universel, inversion, Jacotot, thèse freudienne, Utopie politique

Keywords : Freudian thesis, hypothesis of inversion, Jacotot, Political utopia, sexual ambivalence, universal teaching project

\section{AUTEUR}

\section{ALAIN VERGNIOUX}

Université de Caen 